PROCEEDINGS OF THE

AMERICAN MATHEMATICAL SOCIETY

Volume 125, Number 3, March 1997, Pages 941-942

S 0002-9939(97)03989-0

\title{
COMMENSURABILITY OF FUCHSIAN GROUPS AND THEIR AXES
}

\author{
JAMES W. ANDERSON AND ALAN W. REID
}

(Communicated by Dennis A. Hejhal)

The purpose of this note is to prove the following Theorem. This answers a question raised during the problem session at the Special Session on Geometric Function Theory of the 898th meeting of the AMS in Hartford, CT, the weekend of the fourth of March, 1995, and is related to a question in Mess [4] on whether equality of axis sets for Fuchsian groups implies commensurability.

Theorem. For each arithmetic Fuchsian group $\Gamma$, there exists an infinite order elliptic element e such that $e(\operatorname{ax}(\Gamma))=\operatorname{ax}(\Gamma)$.

Recall that a Fuchsian group is a discrete subgroup of $\mathrm{PSL}_{2}(\mathbf{R}) \cong \operatorname{isom}\left(\mathbf{H}^{2}\right)$. We denote by $\operatorname{ax}(\Gamma)$ the set of axes of hyperbolic elements of the Fuchsian group $\Gamma$.

The proof follows easily from known properties of arithmetic Fuchsian groups. Recall that an arithmetic Fuchsian group is constructed as follows (see [5]). Let $k$ be a totally real number field, and $A$ a quaternion algebra over $k$ which is ramified at all places of $k$ except one, which we may take to be the identity. Let $\rho$ be the embedding of $A$ into $M_{2}(\mathbf{R})$ induced by splitting $A$ at the identity place of $k$. Let $\mathcal{O}$ be an order of $A$, and $\mathcal{O}^{1}$ the elements of reduced norm one in $\mathcal{O}$. Then $\rho\left(\mathcal{O}^{1}\right)$ is a discrete subgroup of $\mathrm{SL}_{2}(\mathbf{R})$ and its projection to $\mathrm{PSL}_{2}(\mathbf{R})$ via $P$ is a finitely generated Fuchsian group of the first kind. A Fuchsian group $\Gamma$ is arithmetic if it is commensurable with some such group $P \rho\left(\mathcal{O}^{1}\right)$.

Subgroups $\Gamma_{1}$ and $\Gamma_{2}$ of $\mathrm{PSL}_{2}(\mathbf{R})$ are commensurable if $\Gamma_{1} \cap \Gamma_{2}$ has finite index in both $\Gamma_{1}$ and $\Gamma_{2}$. Define the commensurator $\operatorname{comm}(\Gamma)$ of a subgroup $\Gamma$ of $\operatorname{PSL}_{2}(\mathbf{R})$ by

$$
\operatorname{comm}(\Gamma)=\left\{\varphi \in \mathrm{PSL}_{2}(\mathbf{R}) \mid \varphi \Gamma \varphi^{-1} \text { is commensurable to } \Gamma\right\} .
$$

It is a theorem of Margulis [3] that $\operatorname{comm}(\Gamma)$ is non-discrete if and only if $\Gamma$ is arithmetic. In particular, since it is non-elementary and non-discrete, $\operatorname{comm}(\Gamma)$ must contain an infinite order elliptic $e[2]$. It is easy to verify that, if $\Gamma_{1}$ and $\Gamma_{2}$ are commensurable, then $\operatorname{ax}\left(\Gamma_{1}\right)=\operatorname{ax}\left(\Gamma_{2}\right)$. Since $e \in \operatorname{comm}(\Gamma), e \Gamma e^{-1}$ and $\Gamma$ are commensurable, and so $\operatorname{ax}(\Gamma)=\operatorname{ax}\left(e \Gamma e^{-1}\right)=e(\operatorname{ax}(\Gamma))$.

In fact, the Theorem holds true for any arithmetic subgroup $\Gamma$ of isom $\left(\mathbf{H}^{2 n}\right)$. Briefly, for such groups, Margulis' result still holds, and Abikoff and Haas [1] have shown that a non-elementary subgroup of isom $\left(\mathbf{H}^{2 n}\right)$ which does not keep a proper subspace invariant is discrete if and only if it contains no infinite order elliptic elements. Since $\Gamma$ arithmetic implies $\mathbf{H}^{2 n} / \Gamma$ has finite volume, $\Gamma$ cannot keep

Received by the editors January 25, 1996.

1991 Mathematics Subject Classification. Primary 20H10.

This research was supported by The Royal Society. 
invariant a proper totally geodesic subspace of $\mathbf{H}^{2 n}$, and therefore the proof follows as before.

The authors would like to thank the University of Aberdeen for its hospitality, and for providing the chance for conversations that led to this short note.

\section{REFERENCES}

1. Wm. Abikoff and A. Haas, 'Nondiscrete groups of hyperbolic motions', Bull. L. M. S. 22 (1990), 233-238. MR 91b:22013

2. T. Jørgensen, 'A note on subgroups of $\mathrm{SL}_{2}(\mathbf{C})$ ', Quart. J. Math. Oxford 28 (1977), 209-212. MR 56:3186

3. G. A. Margulis, Discrete subgroups of semi-simple Lie groups, Springer-Verlag, 1991. MR 92h:22021

4. G. Mess, 'Fuchsian groups with the same simple axes', preprint, 1990.

5. M-F. Vignéras, Arithmétique des algébras de Quaternions, Lecture Notes in Mathematics, vol. 800, Springer-Verlag, 1980. MR 82i: 12016

Faculty of Mathematical Studies, University of Southampton, Highfield, SouthampTON SO17 1BJ ENGLAND

E-mail address: jwa@maths.soton.ac.uk

Department of Mathematics, University of Texas, Austin, Texas 78712

E-mail address: areid@math.utexas.edu 